\title{
TU/e EmonOWEN

\section{Multi-state optical flip-flop memory based on ring lasers coupled through the same gain medium}

\section{Citation for published version (APA):}

Zhang, S., Lenstra, D., Liu, Y., Ju, H., Li, Z., Khoe, G. D., \& Dorren, H. J. S. (2007). Multi-state optical flip-flop memory based on ring lasers coupled through the same gain medium. Optics Communications, 270(1), 85-95. https://doi.org/10.1016/j.optcom.2006.08.031

DOI:

10.1016/j.optcom.2006.08.031

Document status and date:

Published: 01/01/2007

\section{Document Version:}

Publisher's PDF, also known as Version of Record (includes final page, issue and volume numbers)

\section{Please check the document version of this publication:}

- A submitted manuscript is the version of the article upon submission and before peer-review. There can be important differences between the submitted version and the official published version of record. People interested in the research are advised to contact the author for the final version of the publication, or visit the $\mathrm{DOI}$ to the publisher's website.

- The final author version and the galley proof are versions of the publication after peer review.

- The final published version features the final layout of the paper including the volume, issue and page numbers.

Link to publication

\section{General rights}

Copyright and moral rights for the publications made accessible in the public portal are retained by the authors and/or other copyright owners and it is a condition of accessing publications that users recognise and abide by the legal requirements associated with these rights.

- Users may download and print one copy of any publication from the public portal for the purpose of private study or research.

- You may not further distribute the material or use it for any profit-making activity or commercial gain

- You may freely distribute the URL identifying the publication in the public portal.

If the publication is distributed under the terms of Article 25fa of the Dutch Copyright Act, indicated by the "Taverne" license above, please follow below link for the End User Agreement:

www.tue.nl/taverne

Take down policy

If you believe that this document breaches copyright please contact us at:

openaccess@tue.nl

providing details and we will investigate your claim. 


\title{
Multi-state optical flip-flop memory based on ring lasers coupled through the same gain medium
}

\author{
S. Zhang ${ }^{\text {a,* }}$, D. Lenstra ${ }^{\text {b,c }}$, Y. Liu ${ }^{\text {a }}$, H. Ju ${ }^{\text {a }}$, Z. Li ${ }^{\text {a }}$, G.D. Khoe ${ }^{\text {a }}$, H.J.S. Dorren ${ }^{\text {a }}$ \\ ${ }^{a}$ Electro-Optical Communications Group, Eindhoven University of Technology, P.O. Box 513, 5600 MB Eindhoven, The Netherlands \\ ${ }^{\mathrm{b}}$ Opto-Electronic Devices Group, Eindhoven University of Technology, P.O. Box 513, 5600 MB Eindhoven, The Netherlands \\ ${ }^{\mathrm{c}}$ Department of Physics and Astronomy, Vrije Universiteit, FEW, De Boelelaan 1081, 1081 HV Amsterdam, The Netherlands
}

Received 4 May 2006; received in revised form 9 August 2006; accepted 22 August 2006

\begin{abstract}
We investigate a system consisting of multiple ring lasers coupled by a single gain medium. All the ring lasers share a common feedback arm. The output power of an individual laser shows periodic oscillations as a function of time. The periodicity of the oscillation is determined by the ratio of the roundtrip times of the feedback arm and the ring cavity. In the case that two of such ring lasers are coupled, either their oscillation periodicities are synchronized, or the system is bi-stable. In the latter operation regime, the system can act as an optical flip-flop memory whose state be switched by injection of external light. The concept can be extended to multi-state operations; an eight-state optical flip-flop memory is experimentally demonstrated.
\end{abstract}

(C) 2006 Elsevier B.V. All rights reserved.

Keywords: Optical bi-stability; Optical multi-stability; Flip-flop memories; Coupled lasers; Optical feedback; Limit-cycle oscillators; Synchronization; Semiconductor optical amplifiers; Ring laser

\section{Introduction}

Optical bi-stabilities in laser systems have received much attention shortly after the first laser was invented [1,2] and have been well explored using various types of lasers, such as gas lasers [3,4] solid-state lasers [5] dye lasers [6] and semiconductor lasers [7]. There are several types of optical bi-stability: absorptive bi-stability $[1,2,8]$; dispersive bi-stability [9]; two-mode bi-stability $[10,11]$ and bi-stability based on symmetrically coupled nonlinear elements $[12,13]$.

Optical flip-flop memories are optical bi-stable elements with optical set and reset operation. Only a few optical flipflop memory concepts allow extension to multi-state operation. An example is given in [14], which presents a five-state optical flip-flop memory with $\mathrm{CW}$ output, containing five cascaded lasers. Extension of such a memory to more states

\footnotetext{
* Corresponding author. Tel.: +31 40247 5066; fax: +31 402455197. E-mail address: S.Zhang@tue.nl (S. Zhang).
}

is possible, but the power consumption scales linearly with the extension, since every laser has its own active element.

In this paper, we present a multi-state optical flip-flop memory which contains only one active element. This optical system contains a large number of ring lasers, which are coupled together through a single active element and share one common feedback arm. The output power of any individual ring laser shows periodic oscillation as a function of time. In this sense, each individual ring laser behaves as a limit-cycle oscillator in the context of general nonlinear dynamics [15]. (It should be noted that lasers are traditionally called oscillators, which indicate the oscillation of the electromagnetic field at optical frequency. While the concept of oscillators in this paper means optical systems whose output optical power are not continuous but oscillating.) If a large number of lasers are coupled through the same gain medium, we find that the oscillators tend to synchronize only if the oscillation periodicities are sufficiently close to each other; otherwise, the system exhibits a winner-take-it-all multi-stability. Switching between the 
multiple states can be realized by injecting external light to the cavity so that the system can act as a multi-state optical flip-flop memory.

In this paper, we first discuss the dynamic behavior of a single unidirectional ring laser with incoherent feedback. We show that, depending on the control parameters, in particular the feedback strength, the output of the laser exhibits either periodic (limit-cycle type) oscillations or irregular (chaotic-type) oscillations. The periodicity of the limit-cycle oscillator is determined by the ratio of the roundtrip times of the feedback arm and the ring cavity. After having discussed the physics of a single ring laser with feedback, we will investigate the dynamics of a system that consists of two coupled ring lasers sharing the same active element as well as the same feedback arm. We also show that the two limit-cycle oscillators tends to synchronize if their periodicities are close, otherwise the system is bi-stable.

We investigate the underlying physics of the bi-stability and show that different periodicities of two limit-cycle oscillators would modulate the gain in such a way that the roundtrip conditions for the two ring lasers cannot be simultaneously satisfied. Therefore, depending on the initial power, only one oscillator can be active at a time. Furthermore, it is shown that this principle can be straightforwardly extended to a larger number of oscillators sharing the same gain medium. We give experimental evidence supported by numerical simulations for a system that has eight-stable states. Switching between the states can be realized by injection of external light at the wavelength of the selected cavity.

\section{Dynamic behavior of a unidirectional ring laser with incoherent feedback}

Fig. 1 shows the schematic of a unidirectional ring laser with incoherent feedback. A semiconductor optical amplifier (SOA) acts as the gain element. The system consists of a ring cavity and a feedback arm. The ring cavity contains an arrayed waveguide grating (AWG) [16], which acts as a filter to select the lasing wavelength, and an optical isolator that allows the light to propagate in only one direction (thus to ensure unidirectional lasing). The mechanical switch in the ring cavity is used to close or open the cavity

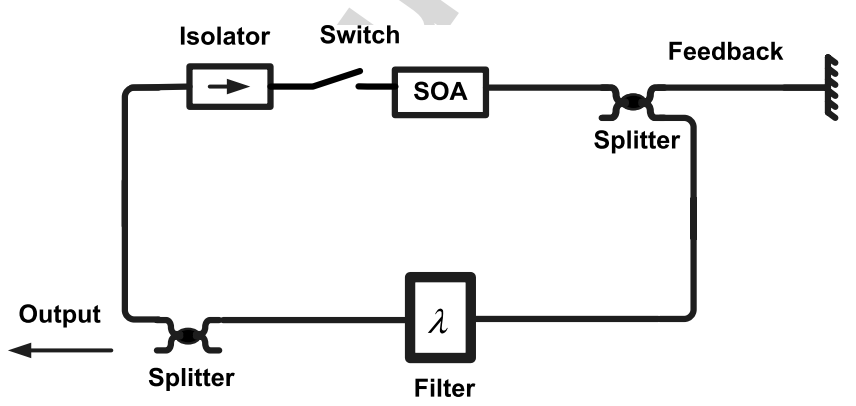

Fig. 1. Schematic of a unidirectional ring laser with feedback. SOA: semiconductor optical amplifier. at demand. A fraction of the lasing light is coupled out of the ring into the feedback arm by a splitter, through which light is reflected back into the SOA from the feedback arm. Note that the feedback light is amplified by the SOA, but it cannot build-up into a lasing state due to the presence of the isolator. The feedback light saturates the gain of the active element and thus influences the lasing light indirectly. Therefore this kind of feedback is called incoherent feedback, in contrast to lasers with conventional optical feedback, in which the feedback light becomes a part of the lasing light when feedback into the laser cavity [17].

The system was built up with fiber-pigtailed components, corresponding to a ring cavity of about $10 \mathrm{~m}$ long. A simple roundtrip equation model describing the system depicted in Fig. 1 is presented in Ref. [18]. In the case of such a long ring cavity and without feedback arm, the model predicts irregular intensity. However, the corresponding experimental results show the lasing light in the long ring cavity to converge on a continuous wave $(\mathrm{CW})$. We conjecture that it is due to the optical filter placed in the ring cavity that the instability has been suppressed. Therefore, an optical filter will be included in the model, and this indeed yields the stable $\mathrm{CW}$ operation as observed. Another modification with the model presented in Ref. [18] is that in that model the reflection was attributed to an SOA facet. In this paper, we extend the model to account for more general reflections.

The optical filter used in our experiments is an AWG [16], which is a planar lightwave circuit (PLC) with one input and eight output ports, as shown in Fig. 2a. It comprises an input aperture, an arrayed waveguide and an output aperture. When multiplexed optical signals are incident on the input aperture, they are spread by diffraction over the arrayed waveguides. Since adjacent waveguides of the array differ in optical length by a fixed amount $\Delta L$, passing through the arrayed waveguides induces phase differences, in such a way that the focus which occurs at the end of the output aperture at different points depends on the wavelength. Thus signals of distinct wavelengths can be selected by disposing the output waveguides at their respective focal points. The corresponding amplitude response of the filter is shown in Fig. 2b. Therefore, as a simple model in Ref. [19], the output optical field $E(t)$ of the AWG filter at a certain wavelength can be expressed as

$E(t)=\sum_{j=0}^{7} r_{j} E(t-\Delta T-j \times \Delta t) \quad(j=0,1, \ldots, 7)$,

where $j$ labels the corresponding waveguide (there are eight of them), $r_{j}$ is the filter coefficient for the waveguide, $\Delta T$ is the propagation time for the shortest waveguide, $\Delta t$ is the propagation time interval corresponding to the length difference $(\Delta L)$ of two adjacent waveguides.

Through including the AWG filter and removing the reflection of the SOA's facet, the roundtrip equations derived in Ref. [18] for the laser field formulated at a reference point inside the SOA can now be expressed as 
a
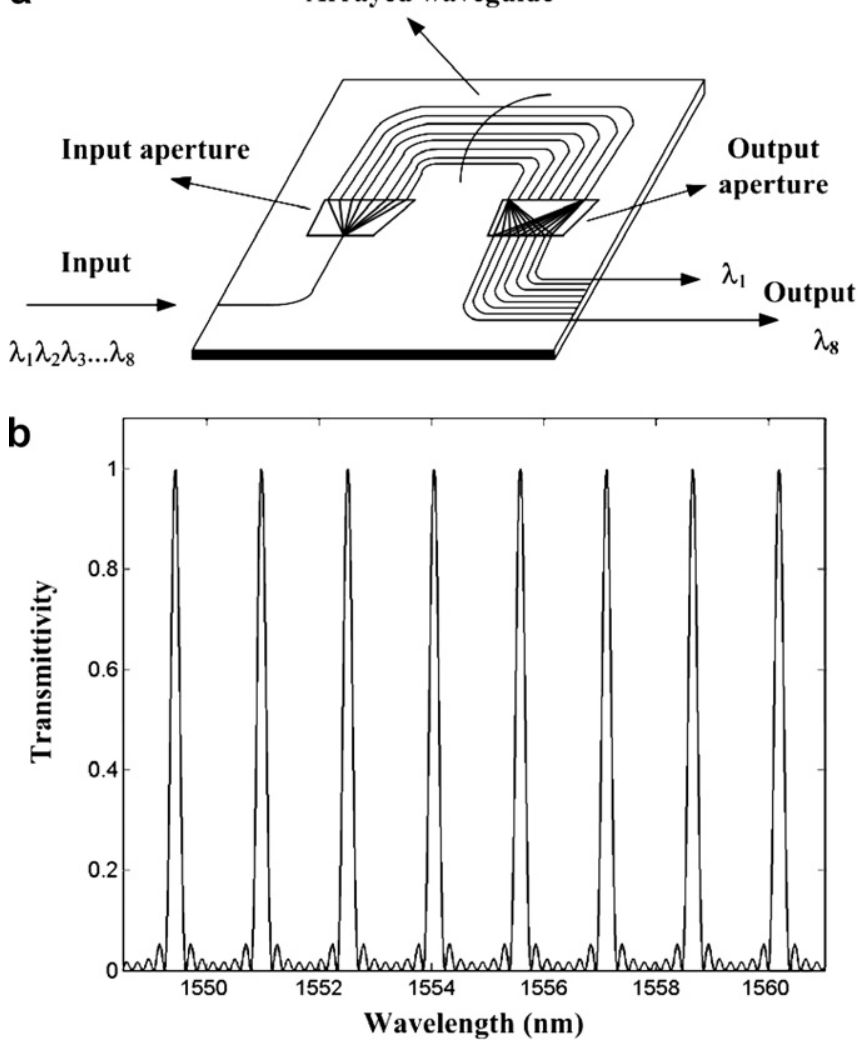

Fig. 2. Panel a: Schematic configuration of the AWG filter; Panel b: Amplitude response of the filter.

$E\left(t+\tau_{\mathrm{r}}\right)=\exp \left[\frac{(1+\mathrm{i} \alpha) \xi L_{\mathrm{s}}}{2 v_{\mathrm{g}}(1+\varepsilon S(t))} n(t)\right] E(t)$,

$E(t)=\sum_{j=0}^{7} r_{j} E(t-j \Delta t) \quad(j=0,1, \ldots, 7)$,

$E_{\mathrm{f}}\left(t+\tau_{\mathrm{f}}\right)=K_{\mathrm{f}} \exp \left[\frac{(1+\mathrm{i} \alpha) \xi L_{\mathrm{s}}}{2 v_{\mathrm{g}}(1+\varepsilon S(t))} n(t)+\mathrm{i} \Psi_{\mathrm{f}}\right] E(t)$,

$$
\begin{aligned}
\frac{\mathrm{d} n(t)}{\mathrm{d} t}= & \frac{I}{q}-\frac{I_{\mathrm{th}}}{q}-\frac{n(t)}{\tau_{\mathrm{c}}} \\
& -\left[\frac{\xi}{(1+\varepsilon S(t))} n(t)-2 v_{\mathrm{g}} \frac{\ln \left(t_{\mathrm{r}}\right)}{L_{\mathrm{s}}}\right]\left(|E(t)|^{2}+\left|E_{\mathrm{f}}(t)\right|^{2}\right),
\end{aligned}
$$

$\Psi_{\mathrm{f}}=\varphi_{\mathrm{f}}-\frac{2 \pi c}{\lambda} \tau_{\mathrm{ext}}-\varphi_{\mathrm{r}}+\frac{2 \pi c}{\lambda} \tau_{\mathrm{r}}, \quad K_{\mathrm{f}}=\frac{\left|t_{\mathrm{f}}\right|}{\left|t_{\mathrm{r}}\right|}$.

In Eqs. (2)-(6), $E(t)$ describes the slowly varying complex optical field amplitude in the ring cavity (but monitored in the SOA) as a function of time $t$, and is related to the photon number $S(t)$ and phase $\phi(t)$ through the wellknown relationship $E(t)=\sqrt{S(t)} \exp [\mathrm{i} \phi(t)]$. Eq. (3) describes the AWG filter explained in Eq. (1). For the sake of simplicity, $r_{0-7}$ has an equal value of 0.125 (1/8). Note that there is no $\Delta T$ in Eq. (3), as it has been included in the roundtrip time of the ring cavity. $E_{\mathrm{f}}(t)$ is the complex optical field amplitude of the feedback light. $\xi$ is the gain coefficient associated with the linearized SOA gain, $\varepsilon$ is the corresponding gain saturation parameter, $\alpha$ is the linewidth enhancement factor, $n(t)$ is the carrier number with respect to its value for the lasing threshold, $L_{\mathrm{s}}$ is the SOA-length, $v_{\mathrm{g}}$ the group velocity of the light in the SOA and $c$ the light speed in vacuum. Furthermore, $\tau_{\mathrm{c}}$ is the carrier lifetime, $\tau_{\mathrm{r}}$ the roundtrip time of the ring cavity and $\tau_{\mathrm{f}}$ the roundtrip time related to the feedback arm. Moreover, $\lambda$ is the wavelength of the lasing light, $I$ the injection current, $I_{\text {th }}$ the threshold current and $q$ the elementary charge unit. Finally, the feedback coefficient $K_{\mathrm{f}}$ is the ratio between the transmission of the feedback arm $\left|t_{\mathrm{f}}\right|$ and the transmission of the ring cavity $\left|t_{\mathrm{r}}\right|$, while the associated phases are $\varphi_{\mathrm{f}}$ and $\varphi_{\mathrm{r}}$, respectively.

The feedback coefficient $K_{\mathrm{f}}$ can be controlled by adjusting the coupling ratio of the splitter connecting the ring cavity and the feedback arm. When $K_{\mathrm{f}}$ is larger than unity, most of the light that outputs the SOA is coupled back into the active element with a certain delay via the feedback arm. Gain saturation induced by the delayed feedback light causes the light in the ring cavity to receive a reduced amplification. Consequently, less light is coupled into the feedback arm so that after one roundtrip of the feedback arm the SOA will be less depleted. As the field evolves further, periodic oscillations of the gain build-up, accompanied by the oscillating intensity of the light, which then has evolved into a periodic (limit-cycle type) oscillation. Fig. 3 shows a particular experimental result in which the output power of the system is periodically oscillating. The result was obtained by using a system made out of fiber-pigtailed components, containing a strained-bulk SOA with an active region of $800 \mu \mathrm{m}$ that was pumped with $190 \mathrm{~mA}$ of current (the threshold current of the system was $120 \mathrm{~mA}$ ).

The simulation shown in Fig. 4 illustrates the evolution process of the oscillation that is visible in Fig. 3. The horizontal axis of Fig. 4 represents the time expressed in nanosecond (lower scale) and in unit of cavity roundtrip time (upper scale). The vertical axes of Fig. $4 \mathrm{a}$ and $\mathrm{b}$ represent the power of the lasing light and the feedback light in the SOA, respectively, while the vertical axis of Fig. 4c represents the net gain in the ring cavity (the amplification of

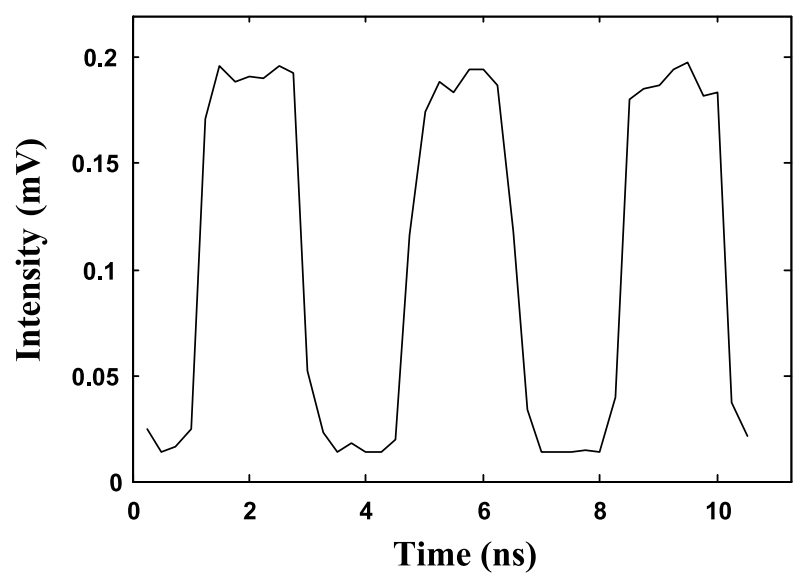

Fig. 3. Typical output of a single ring laser with feedback. 
a Time (Unit: roundtrip time $=50 \mathrm{~ns}$ )
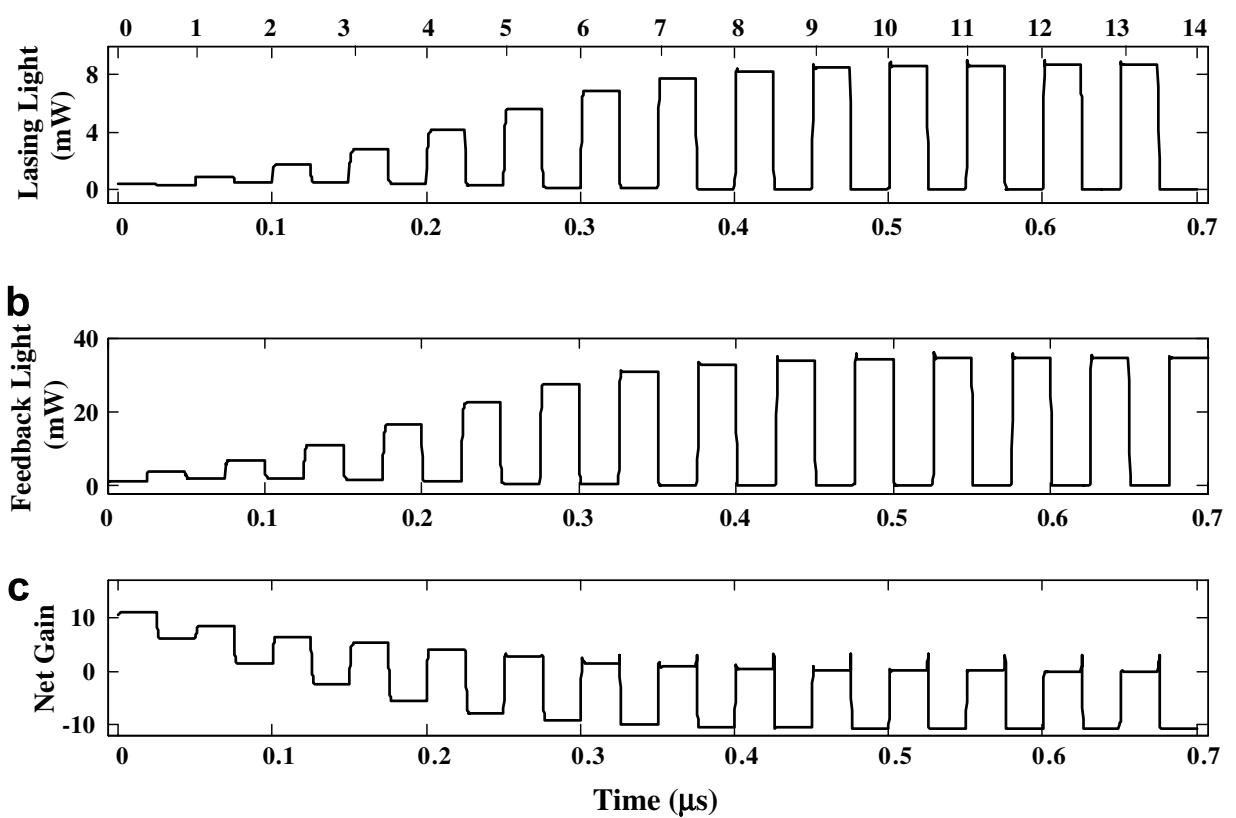

Fig. 4. Simulation of the evolution of the system. Panel a: Power of the lasing light in the SOA versus time; Panel b: Power of the feedback light in the SOA versus time; Panel c: Net gain of the ring cavity versus time.

the SOA minus the loss in the ring cavity). In the simulation, we assume that $K_{\mathrm{f}}=2$ (this means that the transmission of the ring cavity is $50 \%$ compared to the transmission of the feedback arm), $\tau_{\mathrm{r}}$ is $50 \mathrm{~ns}$ (a typical value for our fiber-pigtailed setup) and $\tau_{\mathrm{f}}$ is $25 \mathrm{~ns}$, the values of the other parameters used in the simulations can be found in Table 1.

Suppose that for $t<0$ while the switch in Fig. 1 is open, the SOA is electrically pumped, so that the ring cavity and the feedback arm are filled with spontaneous emission. At $t=0$, the switch is closed and the system starts to evolve. The initial value is chosen such that the laser initially generates $-30 \mathrm{dBm}$ of spontaneous emission around its central frequency. It follows from Fig. $4 \mathrm{a}$ and $\mathrm{b}$ that the light in the ring cavity and the feedback arm receive the same amplification by the SOA. In the first half-roundtrip time, the SOA amplifies the spontaneous emission in both the ring cavity and the feedback arm. Most of the amplified spontaneous emission in the ring cavity is coupled in the feedback

Table 1

Description of value of symbols used in equations

\begin{tabular}{lll}
\hline Symbol & Description & Value \\
\hline$\Gamma$ & Gain confinement factor & 0.14 \\
$\xi$ & Gain coefficient & $3000 \mathrm{~s}^{-1}$ \\
$v_{\mathrm{g}}$ & Group velocity in SOA & $8 \times 10^{7} \mathrm{~m} / \mathrm{s}$ \\
$\alpha$ & Linewidth enhancement factor & 3 \\
$L_{\mathrm{s}}$ & Length of SOA & $800 \mu \mathrm{m}$ \\
$\varepsilon$ & Gain saturation parameter & $1 \times 10^{-7}$ \\
$\Delta t$ & AWG character time & $5 \mathrm{ps}$ \\
$r_{j}$ & AWG filter coefficient & 0.125 \\
$\tau_{\mathrm{c}}$ & Carrier lifetime & $500 \mathrm{ps}$ \\
$q$ & Elementary charge unit & $1.6 \times 10^{-19}$ \\
$I$ & Injection current & $190 \mathrm{~mA}$ \\
$I_{\mathrm{th}}$ & Threshold current & $120 \mathrm{~mA}$ \\
\hline
\end{tabular}

arm during the first half-roundtrip time ( since $K_{\mathrm{f}}=2$ ). The light in the feedback arm is reflected back, and saturates the SOA during the second half-roundtrip time. As a consequence, the light in the ring cavity receives less amplification during the second half-roundtrip time. During the next two roundtrips, the system evolves further and the light in the ring cavity always receives a higher gain during the first half-roundtrip and less gain in the second half-roundtrip. This is due to the fact that the feedback arm has half the length of the ring cavity, and the SOA gain recovery time is small compared to the round-trip time. Thus the power of the light in the ring cavity during each first half-roundtrip time increases with each oscillation periodicity of the roundtrip time $\tau_{\mathrm{r}}$. After several roundtrips, the power of the feedback light has become so strong that the net gain during the second half-roundtrip becomes negative. The lasing light only receives amplification at the corresponding first half of each roundtrip time. Eventually, the system evolves into a stable oscillating state and the net gain is modulated to become periodically negative as shown in Fig. 4c.

In the experiment shown in Fig. 3, we observed that the type of dynamics of the system is determined mainly by two parameters, i.e. the feedback coefficient $K_{\mathrm{f}}$ and the parameter $k=\tau_{\mathrm{f}} / \tau_{\mathrm{r}}$. This is indeed to be expected for a unidirectional ring laser with roundtrip time $\tau_{\mathrm{r}}$, subjected to strong feedback light with delay time $\tau_{\mathrm{f}} . K_{\mathrm{f}}$ represents the feedback strength, and the interplay between the feedback time delay and the roundtrip time is represented by $k$. We will now show the dynamics of the system as a function of $k$, while $K_{\mathrm{f}}$ is kept fixed at the value $K_{\mathrm{f}}=2$.

If $k$ is an integer, the system will evolve into a CW-state. This follows from the fact that for an integer $k$, the length of the feedback arm is an integer times the length of the 


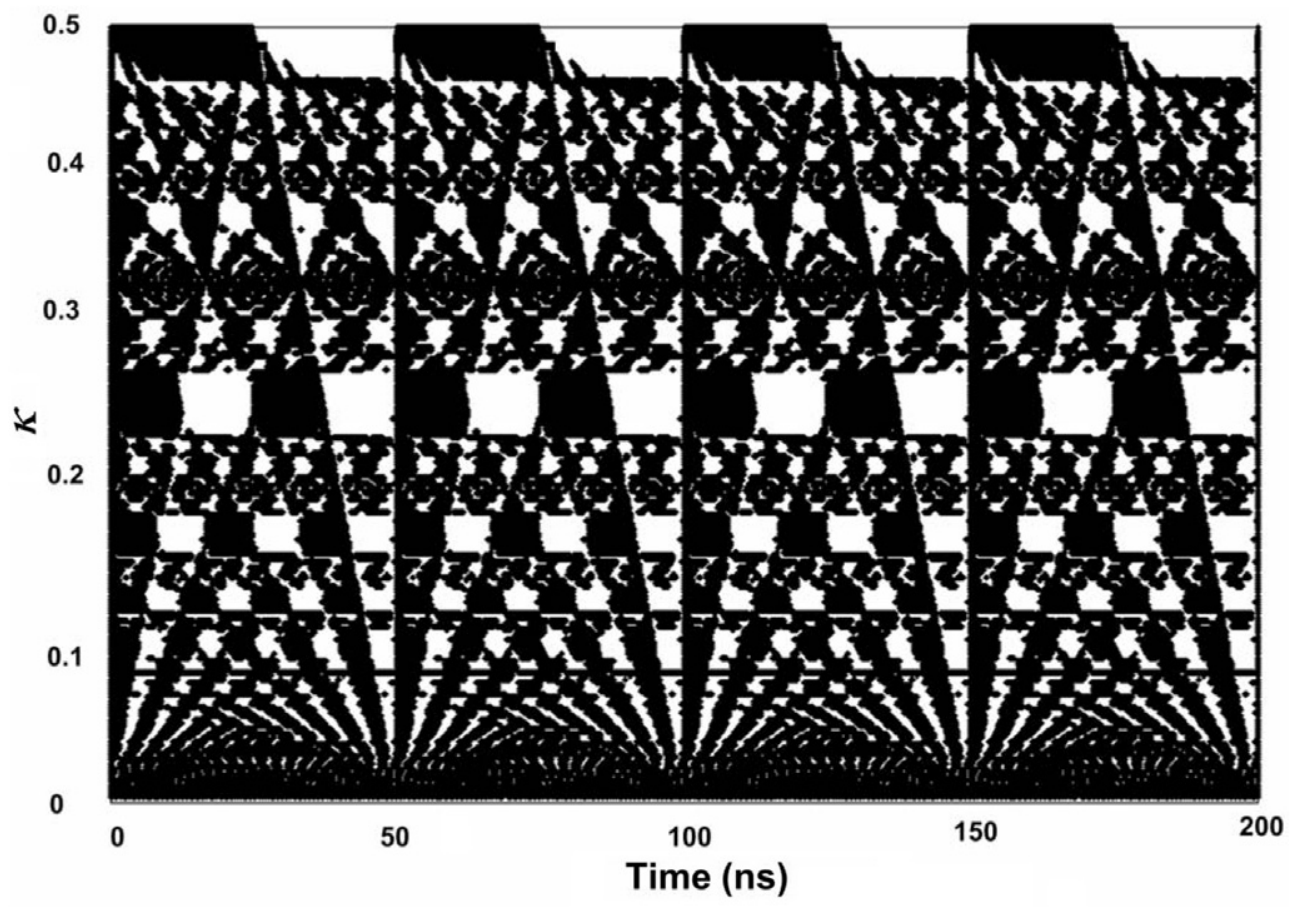

Fig. 5. Overview of the system dynamics for values of $k$ between 0 and 0.5 . For each given value of $k$ the dynamics is indicated horizontally: black areas indicate that the output power of the ring laser is larger than $2.5 \mathrm{~mW}$, whereas white areas indicate that the power is below that value.

ring cavity. This means that the net gain in the ring cavity is constant during each roundtrip and oscillations cannot build-up. However, if $k$ is not an integer, the net gain in the ring cavity fluctuates during each roundtrip due to the imbalance between $\tau_{\mathrm{f}}$ and $\tau_{\mathrm{r}}$, which makes that the system evolves into an oscillation state. This is illustrated in Fig. 5, where on the vertical axis $k$ is varied between 0 and 0.5 , while on the horizontal axis the time is indicated after 100 roundtrip times (so that the transients have died out). The black areas in Fig. 5 indicate that the output power of the ring laser is larger than the average value, while the white areas indicate that the power of the lasing light is below that value. It can be seen in Fig. 5 that the oscillation periodicity is highly sensitive to the value of $k$, whose small change in value may lead to a dramatic change in the oscillation periodicity.

The numerical observation that the oscillation periodicity is highly sensitive to the value of $k$ is confirmed experimentally. A tunable optical fiber delay line, whose length can be adjusted up to a $20-\mathrm{cm}$ range, was inserted into the ring cavity in the setup shown in Fig. 1. The lengths of the ring cavity and the feedback arm were about $10 \mathrm{~m}$ and $2 \mathrm{~m}$, respectively. The time series of the output light was recorded using an oscilloscope. It is shown in Fig. 6a, where 59 oscillations were recorded within $200 \mathrm{~ns}$. The optical fiber delay line was then prolonged to increase the length of the ring cavity by $5 \mathrm{~cm}$, while the remaining setup was kept the same. The time series of the output light with the changed setup is shown in Fig. 6b, where 52 oscillations were observed within $200 \mathrm{~ns}$. It is confirmed in this experiment that the oscillation periodicity is sensitive to the parameter $k$. Furthermore, it is also observed in experiments that the change of the oscillation periodicity is irregular with $k$.

In the dynamic regimes determined by $k$, the dynamics of the system also critically depends on the feedback coefficient $K_{\mathrm{f}}$. To illustrate this, Fig. 7a shows the bifurcation diagram for $S(t)$ as a function of $K_{\mathrm{f}}$ when $k=0.48$. The bifurcation diagram is computed at each value of $K_{\mathrm{f}}$ by firstly letting the system evolve for 100 roundtrip times so as to get rid of transients. Next, the system evolves for another 10 roundtrips, all the values of the output power in the last 10 round-

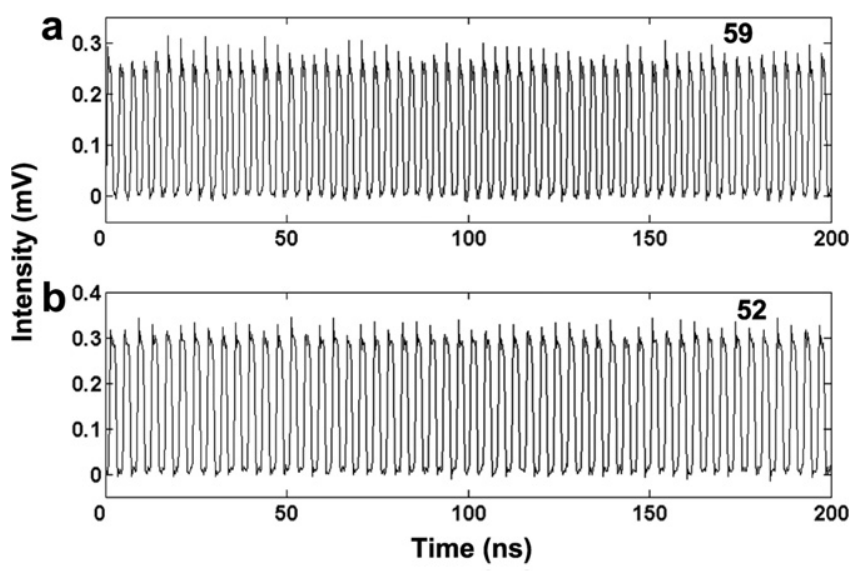

Fig. 6. The change of oscillation periodicity due to a $5-\mathrm{cm}$ change in ring cavity. Panel a: The original oscillation time series (59 periodicities within $200 \mathrm{~ns})$; Panel b: The oscillation time series after the feedback was prolonged $5 \mathrm{~cm}$ ( 52 periodicities within $200 \mathrm{~ns}$ ). 

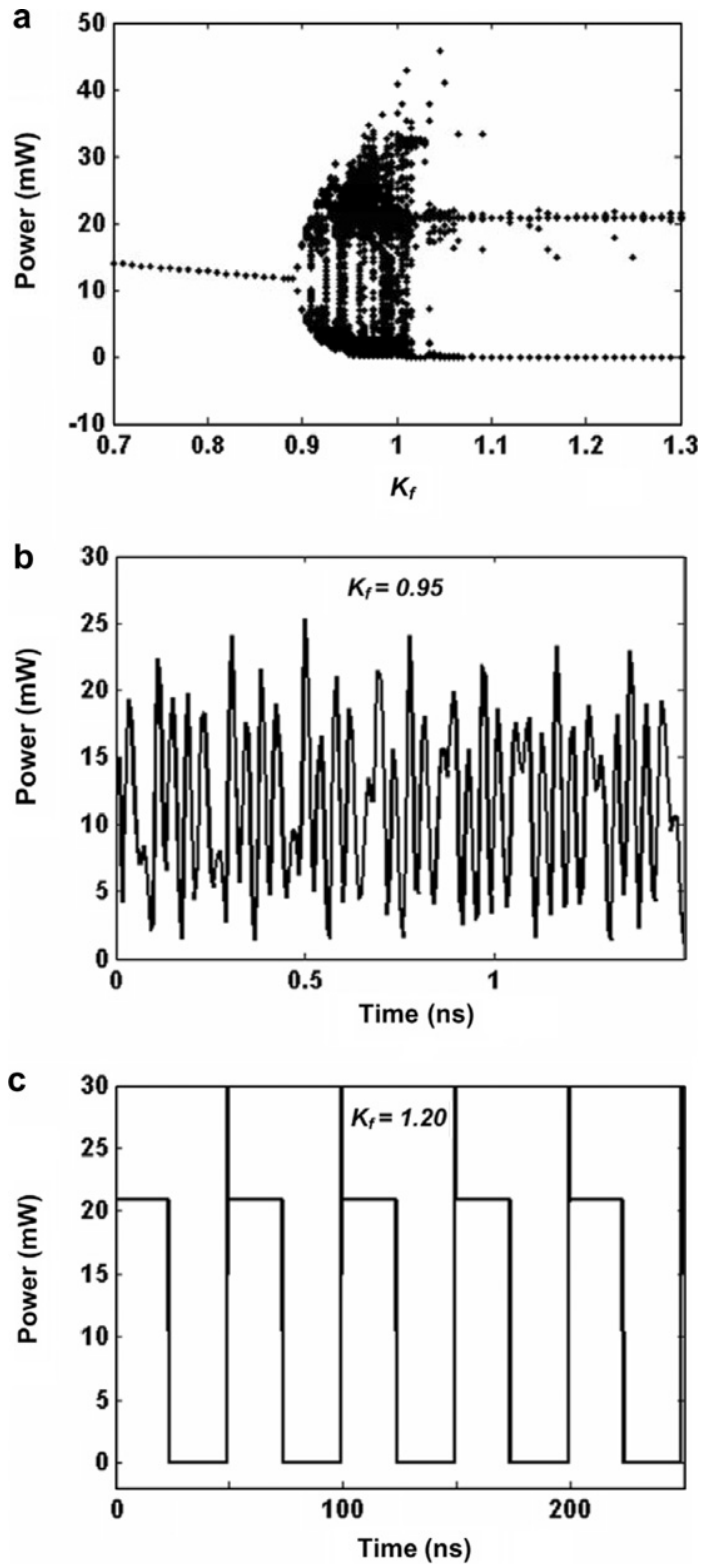

Fig. 7. The system dynamics for $k=0.48$. Panel a: Bifurcation diagram for the laser output power as a function of the $K_{\mathrm{f}}$; Panel b: Time series for $K_{\mathrm{f}}=0.88 ;$ Panel c: Time series for $K_{\mathrm{f}}=1.20$

trips are plotted at regular intervals in the plane that the optical power versus $K_{\mathrm{f}}$. For all values of $K_{\mathrm{f}}$ in the range $[0,0.88]$, the system evolves into $\mathrm{CW}$ operation, although the photon number decreases with increasing $K_{\mathrm{f}}$ due to the enhanced depletion of the SOA. At $K_{\mathrm{f}}=0.88$, a bifurcation, which means a qualitative change in dynamic behavior as a control parameter is smoothly varied, is seen to take place and the system starts to oscillate. When $K_{\mathrm{f}}$ has reached 0.95 , the system shows irregular oscillations as shown in Fig. 7b. If $K_{\mathrm{f}}$ is increased beyond 1.08, the oscillation becomes regular again and finally, this system enters to a limit-cycle regime, as shown in Fig. $7 \mathrm{c}\left(K_{\mathrm{f}}=1.20\right)$.

It is shown in Fig. 5 that for some values of $k$ (for example $k=0.33$ ), the oscillation frequency increases dramati- cally. We will now show how $K_{\mathrm{f}}$ determines the dynamic behavior of the system for the case $k=0.33$. Fig. 8 shows the corresponding bifurcation diagram as a function of $K_{\mathrm{f}}$ when $k=0.33$. It is clear that a bifurcation occurs around $K_{\mathrm{f}}=0.93$ and the system becomes irregular for $K_{\mathrm{f}}$ greater than 0.93 . This indicates the system cannot evolve into a stationary state with larger $K_{\mathrm{f}}$.

To conclude this section, it has been shown that the system with one single ring can exhibit various types of dynamics depending on the combination of the control parameters. When $K_{\mathrm{f}}$ is sufficiently large, the system will operate in the limit-cycle regime for most cases, while the periodicity of the corresponding limit-cycle oscillator can be controlled by the parameter $k$ (as shown in Fig. 5). Once the limit-cycle oscillator has become stationary, i.e. after the transient has died out, the gain of the active element is modulated by the corresponding periodically oscillating light (as shown in Fig. 4c).

\section{Synchronization or bi(multi)-stability of coupled ring lasers with incoherent feedback}

The schematic in Fig. 9 shows two ring lasers that share the same active element and the same feedback arm. We will show in this section that if each individual laser operates in the limit-cycle regime and the losses in both cavities are similar, the limit-cycle oscillators coupled through the same active element synchronize into a same periodicity if their periodicities are sufficiently close to each other, otherwise the oscillators show bi-stability, i.e. one of the lasers is on, while the other one is suppressed. In the remainder of this section, we will investigate this phenomenon and support our discussion with experimental results. A model that describes the system shown in Fig. 9 is given by

$E_{m}\left(t+\tau_{\mathrm{r} m}\right)=\exp \left[\frac{(1+\mathrm{i} \alpha) \xi L_{\mathrm{s}}}{2 v_{\mathrm{g}}(1+\varepsilon S(t))} n(t)\right] E_{m}(t) \quad(m=1,2)$,

$E_{m}(t)=\sum_{j=0}^{7} r_{j} E_{m}(t-j \Delta t) \quad(j=0,1, \ldots, 7)$,

$$
\begin{aligned}
E_{\mathrm{f}}\left(t+\tau_{\mathrm{f}}\right)= & K_{\mathrm{f}} \exp \left[\frac{(1+\mathrm{i} \alpha) \xi L_{\mathrm{s}}}{2 v_{\mathrm{g}}(1+\varepsilon S(t))} n(t)\right] \\
& \times\left[E_{1}(t) \exp \left(\mathrm{i} \Psi_{\mathrm{f}_{1}}\right)+E_{2}(t) \exp \left(\mathrm{i} \Psi_{\mathrm{f}_{2}}\right)\right],
\end{aligned}
$$

$\frac{\mathrm{d} n(t)}{\mathrm{d} t}=\frac{I}{q}-\frac{I_{\mathrm{th}}}{q}-\frac{n(t)}{\tau_{\mathrm{c}}}-\left[\frac{\xi}{(1+\varepsilon S(t))} n(t)-2 v_{\mathrm{g}} \frac{\ln \left(t_{\mathrm{r}}\right)}{L_{\mathrm{s}}}\right]$

$$
\times\left(\left|E_{1}(t)\right|^{2}+\left|E_{2}(t)\right|^{2}+\left|E_{\mathrm{f}}(t)\right|^{2}\right),
$$

$\Psi_{\mathrm{f}_{m}}=\varphi_{\mathrm{f}_{m}}-\frac{2 \pi c}{\lambda_{m}} \tau_{\mathrm{ext}}-\varphi_{\mathrm{r} m}+\frac{2 \pi c}{\lambda_{m}} \tau_{\mathrm{r} m}, \quad K_{\mathrm{f}}=\frac{\left|t_{\mathrm{f}}\right|}{\left|t_{\mathrm{r}}\right|}$.

This model is a straightforward extension of the model for a single ring laser presented by Eqs. (2)-(6). All the symbols are the same as those in Section 2. The index $m$ indicates different ring lasers. It should be noted that there is no index for $K_{\mathrm{f}}$ since we assume that the loss in each ring cavity is the same. We have shown in Section 2 that a ring laser 


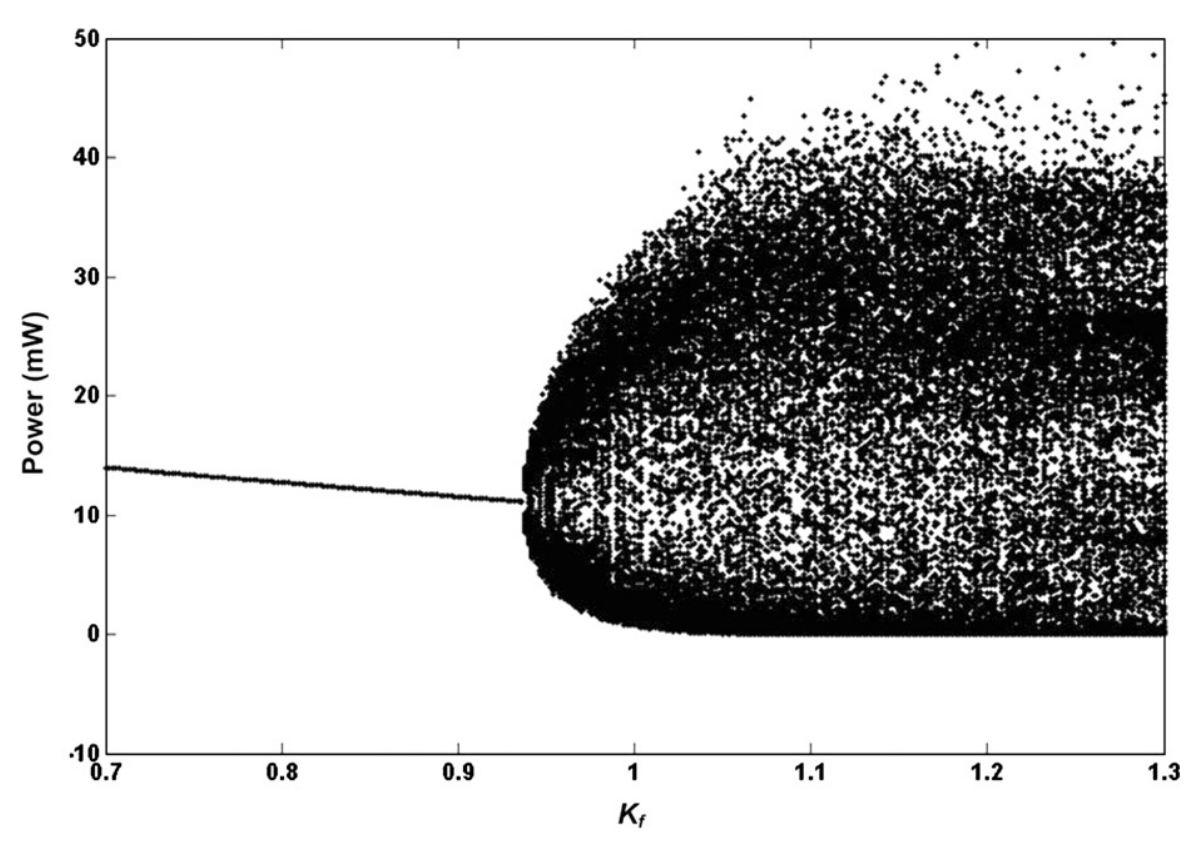

Fig. 8. Bifurcation diagram for the laser output power as a function of $K_{\mathrm{f}}$ for $k=0.33$.

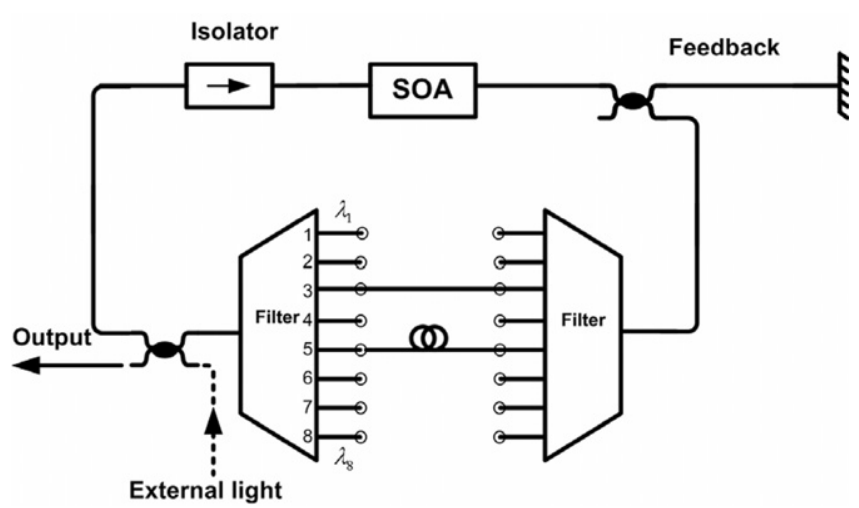

Fig. 9. Schematic of a laser with two ring cavities that share the same active element and the feedback arm.

with feedback can operate in the limit-cycle regime if the feedback strength and the length of the cavities are properly matched. Once the limit-cycle oscillation has built up, the gain of the active element of the ring cavity is modulated by the corresponding periodically oscillating light. The oscillation periodicity can be controlled by adjusting the parameter $k$. We will now discuss the situation in which two ring lasers are coupled through the same active element while sharing the same feedback arm. Suppose the initial power in one of the cavities, say cavity 1 , is larger. If the fields in both cavities evolve in time, the power of cavity 1 grows stronger, and thus forces the gain of the active element to oscillate in the periodicity of the limit-cycle oscillator of cavity 1 . The net gain becomes negative in both cavities when the strong feedback light of cavity 1 passes through the gain medium. If the periodicities of the two limit-cycle oscillators are sufficiently close to each other, they tend to synchronize into the same periodicity. As a consequence, both the two limit-cycle oscillators build-up simultaneously. On the other hand, if the periodicities of the two limit-cycle oscillators are not close enough, the limit-cycle oscillation in cavity 2 cannot build-up due to the periodic negative gain produced by the limit-cycle oscillator of cavity 1 . Since this reasoning applies as well when cavity 2 would be initially dominant, the system is in fact bi-stable as demonstrated below.

Fig. 10 shows a simulation of the time-evolution of the optical power in both cavities. The length of cavity 1 is $10.00 \mathrm{~m}$, while the length of cavity 2 is $10.80 \mathrm{~m}$. The length of the feedback arm is $2.70 \mathrm{~m}$. Fig. 10a shows the case in which the power $\left(P_{1}\right)$ of cavity 1 is initially set to be larger than the power $\left(P_{2}\right)$ in cavity 2 . It is clearly visible that both $P_{1}$ and $P_{2}$ increase in the first ten roundtrips. $P_{1}$ increases faster than $P_{2}$ due to the different initial conditions. Hence, $P_{1}$ modulates the gain deeper compared to $P_{2}$. The net gain of each cavity (both cavities have the same cavity loss) becomes negative during the time that the gain of the active element is deeply saturated by the strong feedback of $P_{1}$ ( similarly as shown in Fig. 4c). Therefore the limit-cycle oscillator of cavity 2 cannot build-up since both oscillators have different oscillation periodicities (see Fig. 10a). If the power $P_{2}$ is initially set to be larger than $P_{1}$, the limit-cycle oscillator of cavity 2 builds up and the limit-cycle oscillator of cavity 1 is eventually suppressed as illustrated in Fig. 10b.

This numerical result is verified experimentally by implementing the scheme of Fig. 9 as follows. Two filters are used as wavelength selective elements. In our experiment, the filters were AWGs, which acted as eight-port filters that were implemented to form the multiple ring cavities. The AWGs used in our setup had a $3 \mathrm{~dB}$ bandwidth of $0.50 \mathrm{~nm}$ at each port. The center wavelengths were in a range between $1549.30 \mathrm{~nm}$ and $1560.20 \mathrm{~nm}$ with a regular 

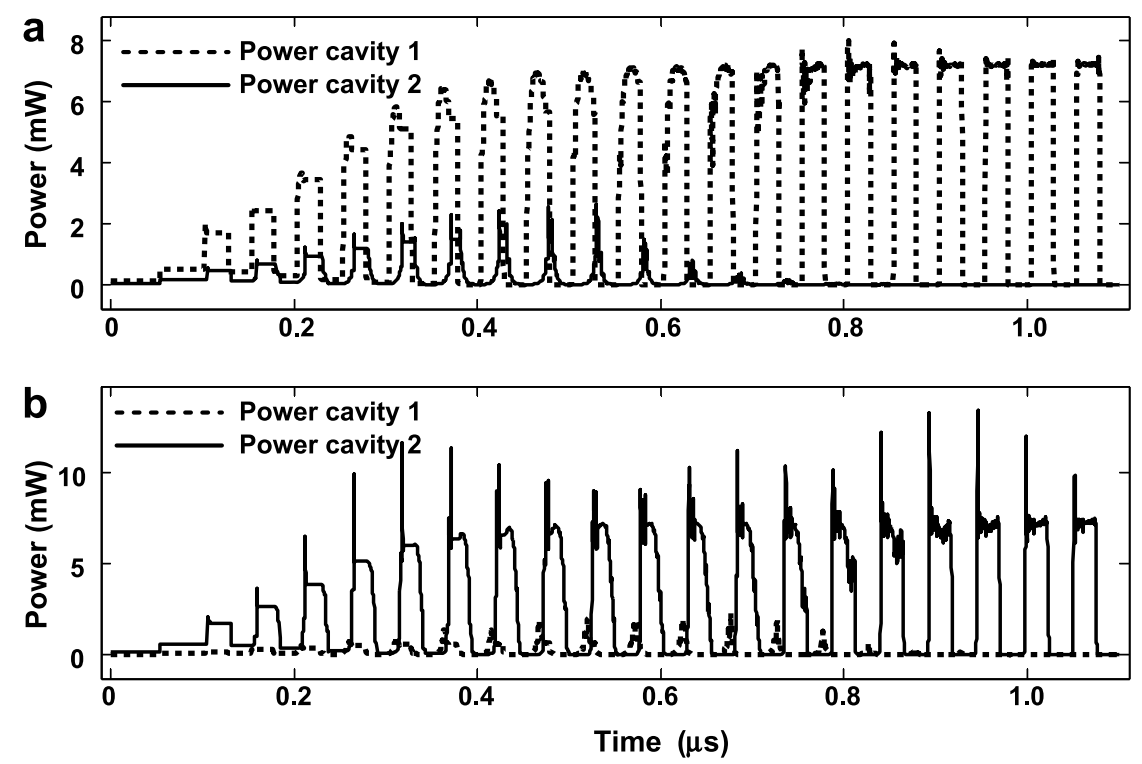

Fig. 10. Simulation result illustrating bi-stable behavior of two coupled ring lasers. The dashed line indicates the power in cavity 1 and the solid line shows the power in cavity 2. Panel a: The initial values are set such that the power in cavity 1 is larger than in cavity 2; Panel b: The initial power in cavity 2 is larger than in cavity 1.

interval of $1.40 \mathrm{~nm}$. The two ports used in this experiment had central wavelengths of $1549.30 \mathrm{~nm}$ and $1558.70 \mathrm{~nm}$, respectively. As indicated in Fig. 9, they were interconnected with short pieces of optical fiber. The laser active element was a strained-bulk SOA with an active length of $800 \mu \mathrm{m}$ that was pumped with $190 \mathrm{~mA}$ of current, which corresponds to a small signal roundtrip gain of $25 \mathrm{~dB}$. The two ring lasers had a threshold current of $120 \mathrm{~mA}$. Under these conditions, the system was set in the limitcycle regime $\left(K_{\mathrm{f}} \approx 2\right)$. Firstly, we established lasing in the individual ring cavity by disconnecting the other. The result is shown in Fig. 11, where the optical spectra of the individual lasers are shown in Fig. 11a and b, the corresponding time series are shown in Fig. 11c and d. It is clearly visible that both individual lasers exhibit limit-cycle behavior. The limit-cycle oscillators had a difference in periodicity of $0.07 \mathrm{~ns}(2.56 \%)$. Secondly, both ring cavities were connected and the output optical spectrum is shown in Fig. 11e. The system now operated simultaneously with both limit-cycle oscillators synchronized at the same periodicity, as demonstrated in the corresponding time series in Fig. 11f.

We now turn back to the case in which only one laser is lasing. Suppose this is the laser lasing at the wavelength of
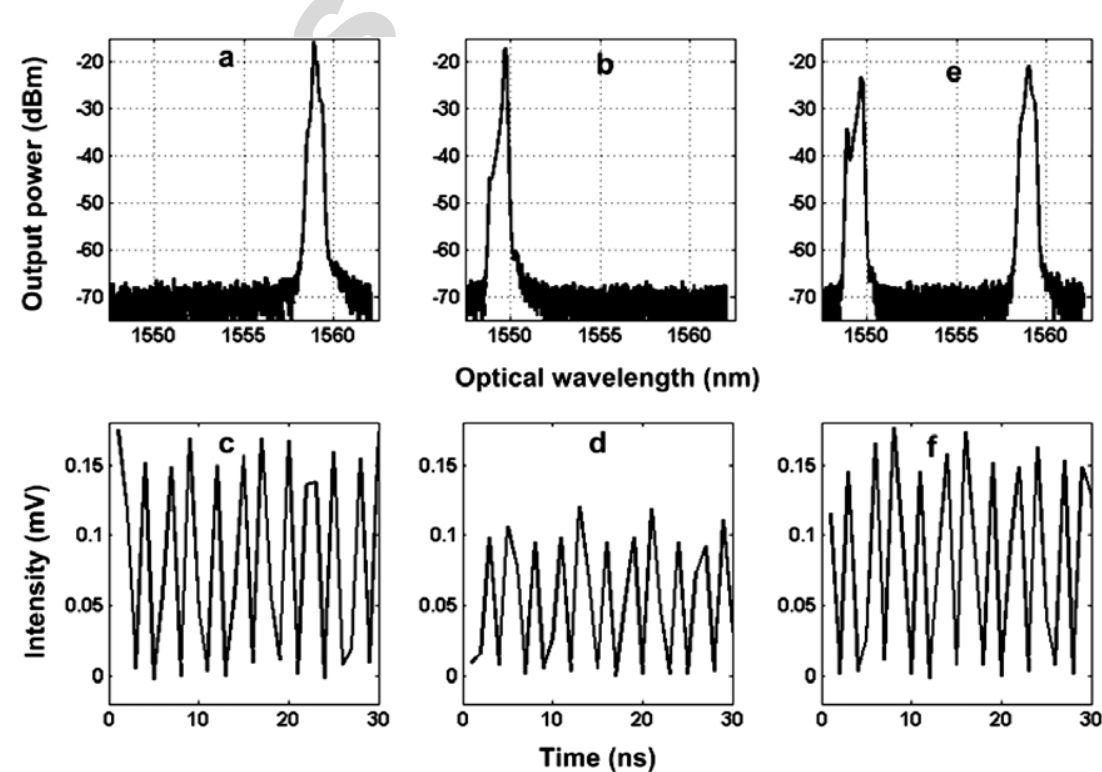

Fig. 11. Synchronization of two coupled oscillators. Panel a: Output spectrum when only the ring cavity of $1558.7 \mathrm{~nm}$ was connected; Panel b: Output spectrum when only the ring cavity of $1549.3 \mathrm{~nm}$ was connected; Panels $\mathrm{c}$ and d: Time series corresponding to the limit-cycle in Panels a and b, respectively; Panel e: The output spectrum when both ring cavities were connected; Panel f: Time series corresponding to Panel e. 

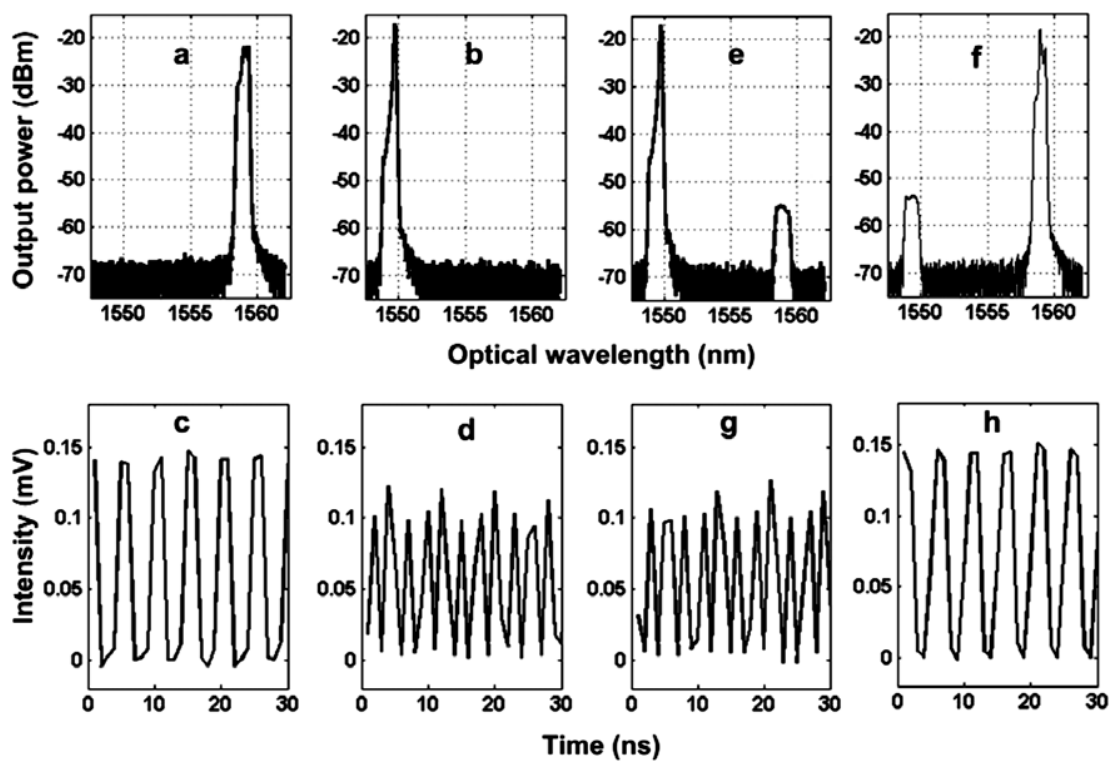

Fig. 12. Bi-stability of two coupled oscillators. Panel a: Optical spectrum for the case that the ring cavity with a central wavelength of $1558.7 \mathrm{~nm}$ was connected (after adding an additional meter fiber into the cavity); Panel b: Output spectrum when only the ring cavity with a central wavelength of $1549.3 \mathrm{~nm}$ was connected; Panels $\mathrm{c}$ and d: Time series corresponding to Panels a and b, respectively; Panel e: Optical spectrum when both ring cavities were connected, with the ring cavity of $1549.3 \mathrm{~nm}$ connected first; Panel f: Same as in Panel e, but now the ring cavity of $1558.7 \mathrm{~nm}$ connected first; Panels g and h: Time series corresponding to Panels e and f, respectively.

$1558.70 \mathrm{~nm}$. If we added an additional meter of fiber in its cavity, while keeping the rest of the experimental setup unchanged, the dynamics of the changed ring laser had dramatically changed as shown in Fig. 12c. Fig. 12a and b shows the optical spectra of the individual cavities, similar as the spectra shown in Fig. 11a and b. The corresponding time series are shown in Fig. 12c and d. It is clearly seen that both cavities still showed limit-cycle behavior, but the difference in periodicity between the two limit-cycle oscillators had increased to $2.29 \mathrm{~ns}(85.71 \%)$. This is due to the fact that the additional meter of fiber had changed the ratio $k$ between the feedback arm and the ring cavity for the ring laser at the wavelength of $1558.70 \mathrm{~nm}$.

We now re-connected both cavities and observed the system to become bi-stable (Fig. 12e-h). In the first state the ring laser at $1549.30 \mathrm{~nm}$ was lasing, while the power in the other cavity was suppressed with $35 \mathrm{~dB}$ as shown in Fig. 12e. The corresponding limit-cycle oscillation is shown in Fig. 12g. In the second state the ring laser at $1558.70 \mathrm{~nm}$ was lasing while the first laser was suppressed as shown in Fig. 12f. The corresponding limit-cycle oscillation is shown in Fig. $12 \mathrm{~h}$.

To induce switching between the states, external light at the same wavelength of the suppressed cavity is injected into the system. The injected light, propagating through the suppressed ring cavity, is amplified by the SOA and starts to build-up an oscillation in the suppressed cavity. As a result of this, the injected light disturbs the modulation of the gain caused by the limit-cycle oscillation in the dominant ring cavity. When the power of the injected light exceeds a threshold value, the gain is forced to follow the periodicity of the limit-cycle oscillator of the laser that is initially suppressed. Eventually, the laser that initially is suppressed becomes dominant. After the system has switched states, it maintains its state even if the injected light is removed. In our experiments, $0.01 \mathrm{~mW}$ of external
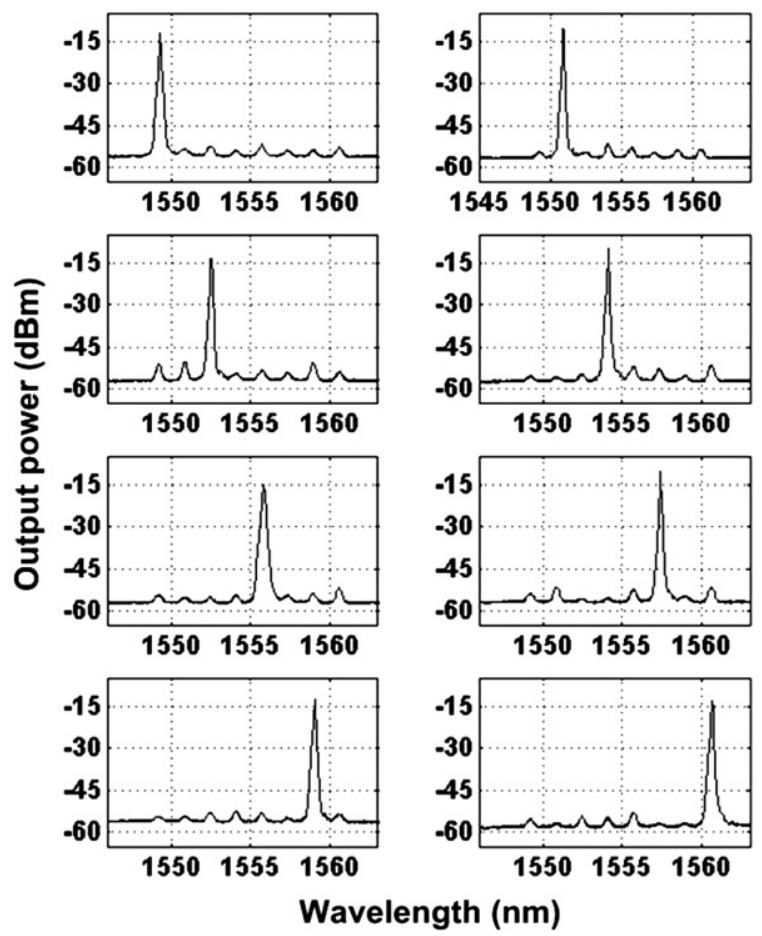

Fig. 13. Output optical spectra for each of the eight states for the eightstable system with eight ring cavities. The switching between these states was realized by injection of external light at the wavelength of the chosen ring cavity. 
light was required to switch the state of the system. In this way, a two state optical flip-flop memory is realized.

When all eight cavities in Fig. 9 were connected, we realized a system of eight ring lasers sharing the same active element and the same feedback arm. If all individual ring lasers were set to operate in the limit-cycle regime, the coupled limit-cycle oscillators behaved in a similar fashion as the above described case of two coupled limit-cycle oscillators. If one limit-cycle oscillator firstly built up first, all the other oscillators either synchronized into the same periodicity or became suppressed. As an extreme example, if no combination of two limit-cycle oscillators could synchronize together, the system showed eight-stability as shown in Fig. 13. The contrast ratio between the dominant and the suppressed states is more than $35 \mathrm{~dB}$. Similar as in the bi-stable case, injection of the external light at the same wavelength of the chosen suppressed ring cavity was used to switch the state of the system.

\section{Conclusion}

In this paper, we have shown that a unidirectional ring laser with incoherent feedback shows various types of dynamic behavior such as $\mathrm{CW}$, periodic (limit-cycle type) oscillation and irregular (chaotic-type) oscillation. Those behavior are determined by the combination of two control parameters: the feedback strength and the ratio between the roundtrip times of the feedback arm and the ring cavity. When two (or more) of such ring lasers, operating in the limit-cycle regime, are coupled together, either their oscillation periodicities are synchronized to have a common oscillation periodicity, or the system shows bi-stability between the lasers. The reason for the bi(multi-)stability is that the strong feedback light causes modulated depletion of the gain of the active element and forces the net gain to become periodically negative, which prohibits the fulfillment of the roundtrip condition for the other lasers. An eight-state optical flip-flop memory has been experimentally demonstrated.

Switching between different states has been realized by injecting external light at the wavelength of the cavity intended to switch on. The switching speed of the flip-flop memory is investigated experimentally and numerically. Numerical results indicate that it takes approximately thirty roundtrips to switch state. This is confirmed by experiments. Decreasing the cavity length is essential to realize higher switching speed. Integration of the system on a single chip would greatly increase the switching speed. The dimensions of an integrated version might be limited by the size of the filter, the active element and the isolator. The dimensions of AWGs and SOAs are typical at the order of several hundreds of micrometers, while the dimensions of the integrated isolators are at the order of millimeters, with losses proportional to its length [20]. Therefore the dimensions of an integrated version of this system is at the order of centimeters, which leads to a switching speed up to a few hundreds MHz. Note that when the roundtrip time of the laser cavity is comparable with or slightly smaller than the gain recovery time, the gain medium is less saturated by the strong feedback light. The net gain of the cavity still becomes periodically negative in the presence of strong feedback light, but the amplitude of the gain modulation is reduced due to gain saturation. The bi-stable phenomenon still exists, but in a narrower bi-stable regime. Numerical simulations indicate that if the cavity length is much shorter than the gain recovery time (for example if the cavity length is $0.1 \mathrm{~mm}$ ) the bi-stability vanishes.

Investigation of this system is interesting, not only since it provides potential applications of optical bi(multi)-stability in engineering, but also it brings new insight in the nature and understanding of coupled oscillators. Coupled oscillators are widely considered to provide insight in collective behavior in physics [21], chemistry [22], biology [23] and even human society [24]. The predominant effect of coupled oscillators with weak coupling is synchronization, which means the oscillators are synchronized in a same oscillation frequency $[25,26]$. With stronger coupling [27] or proper time delay [28,29], a phenomenon called amplitude death, which means that the oscillators cease oscillating, takes place. Although all the coupled oscillators investigated before are different as to the disciplines they belong to, their complexities, their ways of coupling and their dynamic behavior, they have one property in common: each of the coupled oscillators has its own gain source. The optical system presented in this paper is basically a group of limit-cycle oscillators coupling through the same gain source. The coupled oscillators in this system show either synchronization or winner-take-it-all multi-stability behavior. It should be remarked that this synchronization phenomenon is similar to the synchronization of weakly coupled oscillators that have separated gain mediums [30-32]. However, multi-stability between the coupled limit-cycle oscillators seems not has been described before. It is worthwhile to further investigate the quantitative characterization of the synchronization regimes and this coupling method in the context of general nonlinear dynamics.

\section{Acknowledgments}

This work is supported by the Netherlands Organisation for Scientific Research (NWO), the Technology Foundation STW and the Ministry of Economic Affairs through respectively the NRC Photonics grant, the Innovation Research Incentives Scheme programme and Grant ETC. 5579 .

\section{References}

[1] G.J. Lasher, Solid-State Electronics 7 (1964) 707.

[2] M.I. Hathan, J.C. Marinace, R.F. Rutz, A.E. Michel, G.J. Lasher, Journal of Applied Physics 23 (1964) 473.

[3] H.M. Gibbs, S.L. McCall, T.N.C. Venkatesan, Physical Review Letters 36 (1976) 1135.

[4] D. Lenstra, Physics Reports 59 (1980) 300. 
[5] H.M. Gibbs, Optical Bistability: Controlling Light with Light, Academic Press, London, 1985.

[6] R. Roy, L. Mandel, Optics Communications 34 (1980) 133.

[7] H. Kawaguchi, Bistabilities and Nonlinearities in Laser Diodes, Artech House, Boston, 1994.

[8] K. Nonaka, H. Tsuda, T. Kurokawa, H. Uenohara, H. Iwamur, IEEE Photonics Technology Letters 5 (1993) 139.

[9] M.J. Adams, Solidstate Electronics 30 (1987) 43.

[10] C.L. Tang, A. Schremer, T. Fjitia, Applied Physics Letters 51 (1987) 1392.

[11] L. Xu, B.C. Wang, V. Baby, I. Glesk, P.R. Proucnal, IEEE Photonics Technology Letters 14 (2002) 149.

[12] M.T. Hill, H. de Waardt, G.D. Khoe, H.J.S. Dorren, IEEE Journal of Quantum Electronics 37 (2001) 405.

[13] M.T. Hill, H.J.S. Dorren, T. de Vries, X.J.M. Leijtens, J.H. den Besten, B. Smalbrugge, Y.S. Oei, H. Binsma, G.D. Khoe, M.K. Smit, Nature 432 (2004) 206.

[14] S. Zhang, D. Owens, Y. Liu, M.T. Hill, D. Lenstra, A. Tzanakaki, G.D. Khoe, H.J.S. Dorren, IEEE Photonics Technology Letters 17 (2005) 1962.

[15] S.H. Strogatz, Nonlinear Dynamics and Chaos: With Applications to Physics, Biology, Chemistry, and Engineering, Addison-Wesley, Amsterdam, 1994.

[16] M.K. Smit, C. van Dam, IEEE Journal of Select Topics in Quantum Electronics 2 (1996) 236.

[17] G.H.M. van Tartwijk, D. Lenstra, Journal of Optics B. Quantum and Semiclassical Optics Opt. 7 (1995) 87.
[18] S. Zhang, Y. Liu, D. Lenstra, M.T. Hill, H. Ju, G.D. Khoe, H.J.S. Dorren, IEEE Journal of Select Topics in Quantum Electronics 10 (2004) 1093.

[19] G. Toptchiyski, S. Kindt, K. Petermann, E. Hilliger, S. Diez, H.G. Weber, Journal of Lightwave Technology 17 (1999) 2577.

[20] H. Shimizu, Y. Nakano, Journal of Lightwave Technology 24 (2006) 38.

[21] P. Hadley, M.R. Beasley, Applied Physics Letters 52 (1988) 1619.

[22] I.Z. Kiss, Y. Zhai, J.L. Hudson, Science 296 (2002) 1676.

[23] A.T. Winfree, The Geometry of Biological Time, Springer-Verlag, New York, 1980.

[24] Z. Neda, E. Ravasz, Y. Berchet, T. Vicsek, A.L. Barabasi, et al., Nature 403 (2000) 849.

[25] Y. Kuramoto, Progress of Theoretical Physics Supplement 79 (1984) 223.

[26] S.H. Strogatz, Physica D 143 (2000) 1.

[27] R.E. Mirollo, S.H. Strogatz, Journal of Statistical Physics 60 (1990) 245.

[28] D.V. Ramana Reddy, A. Sen, G.L. Johnston, Physical Review Letters 80 (1998) 5109.

[29] D.V. Ramana Reddy, A. Sen, G.L. Johnston, Physical Review Letters 85 (2000) 3381.

[30] P.C. Matthews, R.E. Mirollo, S.H. Strogatz, Physica D 52 (1991) 293.

[31] L.M. Pecora, Physical Review E 58 (1998) 347.

[32] M. Rosenblum, A. Pikovsky, Contemporary Physics 44 (2003) 401. 